\title{
Decomposition and Intersection of Two Fuzzy Numbers for the Fuzzy
}

\section{Preference Relations}

\section{Hui-Chin Tang}

Department of Industrial Engineering and Management, National Kaohsiung University of Applied Sciences, Kaohsiung 80778, Taiwan, R.O.C.

tang@kuas.edu.tw

\section{Abstract}

In fuzzy decision problems, the ordering of fuzzy numbers is the basic problem. Among which, the fuzzy preference relation is the reasonable one to represent preference relation by a fuzzy membership function. This paper studies the Nakamura's and Kołodziejczyk’s preference relations. Eight cases of representing different level of overlapping between two triangular fuzzy numbers are considered. We analyze the ranking behaviors of all possible combinations of decomposition and intersection of two fuzzy numbers for the Nakamura's and Kołodziejczyk's preference relations of these test cases. The results indicate that the decomposition and intersection can affect the fuzzy preference relations, thereby the final total order relation of fuzzy numbers.

Keywords: fuzzy number; ranking; preference relation. 


\section{Introduction}

For solving decision making problems in a fuzzy environment, the overall utilities of a set of alternatives are represented by the fuzzy sets or fuzzy numbers. A fundamental problem of a decision making procedure involves ranking a set of fuzzy sets or fuzzy numbers. Ranking functions, reference sets and preference relations are three categories to rank a set of fuzzy numbers. For a detailed discussion, we refer the reader to surveys by Chen and Hwang [1] and Wang and Kerre [2, 3]. For ranking a set of fuzzy numbers, this paper concentrates on the fuzzy preference relations which are able to represent preference relations in linguistic or fuzzy terms and to make pairwise comparison. To propose the fuzzy preference relation, Nakamura [4] employed a fuzzy minimum operation followed by the Hamming distance. Kołodziejczyk [5] considered the common part of two membership functions and used the fuzzy maximum and Hamming distance. Yuan [6] compared the fuzzy subtraction of two fuzzy numbers with real number zero and indicated that the desirable properties of a fuzzy ranking method are the fuzzy preference presentation, rationality of fuzzy ordering, distinguishability and robustness. Li [7] included the influence of levels of possibility of dominance. Lee [8] presented a counterexample of Li's method [7] and proposed an additional comparable property. Asady [9] revised the method of Wang et al. [10] based 
on deviation degree. Zhang et al. [11] presented a fuzzy probabilistic preference relation. Zhu et al. [12] proposed the hesitant fuzzy preference relations. Wang [13] adopted the relative preference degrees of the fuzzy numbers over average. Liu et al. [14] modified the Farhadinia's hesitant fuzzy set lexicographical ordering method [15] and was more reasonable in more general cases. This paper evaluates and compares two fundamental fuzzy preference relations, one is proposed by Nakamura [4], and the other is by Kołodziejczyk [5]. The intersection of two membership functions and the decomposition of two fuzzy numbers are two differences between these two preference relations. Since the desirable criteria cannot easily be represented in mathematical forms, their performance measures are often tested by using test examples and judged intuitively. To this end, we consider eight complex cases of representing different level of overlapping between two fuzzy numbers. For the Nakamura's and Kołodziejczyk’s fuzzy preference relations, this paper analyzes and compares the ordering behaviors of the decomposition and intersection tested on a group of selected examples.

The organization of this paper is as follows. Section 2 briefly reviews the fuzzy sets and fuzzy preference relations and presents the eight test cases. Section 3 analyzes the Nakamura's fuzzy preference relation and presents an algorithm. Section 4 presents 
the behaviors of Kołodziejczyk's fuzzy preference relation. Section 5 analyzes the effect of the decomposition and intersection on the fuzzy preference relations. Finally, we end with some concluding remarks.

\section{Fuzzy Sets and Test Problems}

We firstly review the basic notations of fuzzy sets and fuzzy preference relations. Consider a fuzzy set $A$ defined on a universal set of real numbers $\Re$ by the membership function $A(\mathrm{x})$, where $A(x): \Re \rightarrow[0,1]$.

Definition 1. Let $A$ be a fuzzy set. The support of $A$ is the crisp set $S_{A}=\{x \in \mathfrak{R} \mid A(x)>0\} . A$ is called normal when $\sup _{x \in S_{A}} A(x)=1$. An $\alpha-$ cut of $A$ is a crisp set $A_{\alpha}=\{x \in \mathfrak{R} \mid A(x) \geq \alpha\} . A$ is convex if, and only if, each of its $\alpha$-cut is a convex set.

Definition 2. A normal and convex fuzzy set whose membership function is piecewise continuous is called a fuzzy number.

Definition 3. A triangular fuzzy number $A$, denoted $A=(a, b, c)$, is a fuzzy number with membership function given by

$$
A(x)= \begin{cases}\frac{x-a}{b-a} & \text { if } a \leq x \leq b \\ \frac{c-x}{c-b} & \text { if } b \leq x \leq c \\ 0 & \text { otherwise }\end{cases}
$$


where $-\infty<a \leq b \leq c<\infty$. The set of all triangular fuzzy numbers on $\Re$ is denoted by $\mathrm{TF}(\mathfrak{R})$.

Definition 4. For a fuzzy number $A$, the upper boundary set $\bar{A}$ of $A$ and the lower boundary set $\underline{A}$ of $A$ are respectively defined as

$$
\bar{A}(x)=\sup _{y \geq x} A(y)
$$

and

$$
\underline{A}(x)=\sup _{y \leq x} A(y) \text {. }
$$

Definition 5. The Hamming distance between two fuzzy numbers $A$ and $B$ is defined by

$$
\begin{gathered}
\mathrm{d}(A, B)=\int_{R}|A(x)-B(x)| d x \\
=\int_{A(x) \geq B(x)} A(x)-B(x) d x+\int_{B(x) \geq A(x)} B(x)-A(x) d x .
\end{gathered}
$$

Definition 6. Let $A$ and $B$ be two fuzzy numbers. Let $\times$ be an operation on $\Re$, such as $+,-, *, \div \ldots$ By extension principle, the extended operation $\otimes$ on fuzzy numbers can be defined by

$$
\mu_{A \otimes B}(z)=\sup _{x, y: z=x \times y} \min \{A(x), B(y)\} .
$$

Definition 7. A fuzzy preference relation $R$ is a fuzzy binary relation with membership function $R(A, B)$ indicating the degree of preference of fuzzy number $A$ over fuzzy number $B$.

1. $R$ is reciprocal if, and only if, $R(A, B)=1-R(B, A)$ for all fuzzy numbers $A$ and 
$B$.

2. $R$ is transitive if, and only if, $R(A, B) \geq 0.5$ and $R(B, C) \geq 0.5$ implies $R(A, C) \geq 0.5$ for all fuzzy numbers $A, B$ and $C$.

3. $\quad R$ is a fuzzy total ordering if, and only if, $R$ is both reciprocal and transitive.

For simplicity, we denote $R^{\prime}(A, B)$ for the degree of preference of fuzzy number $B$ over fuzzy number $A$.

The evaluation criteria of comparing two fuzzy numbers cannot easily be represented in mathematical forms, it is often tested on a group of selected examples. The membership functions of two fuzzy numbers can be overlapping/nonovelapping, convex/nonconvex, and normal/nonnormal. All the approaches proposed in the literature seem to suffer from some questionable examples, especial for the portion of overlapping between two membership functions.

Let $A\left(a_{1}, b_{1}, c_{1}\right)$ and $B\left(a_{2}, b_{2}, c_{2}\right)$ be two triangular fuzzy numbers. Figure 1 displays eight test cases of representing different level of overlapping between $A$ and $B$ and Table 1 shows the area $Q_{i}$ of $i$-th region in each case. More precisely, the eight test cases are

Case 1. $a_{1} \leq a_{2}, b_{1} \leq b_{2}, c_{1} \leq c_{2}$.

Case 2. $a_{1} \leq a_{2}, b_{1} \geq b_{2}, c_{1} \leq c_{2}$. 
Case 3. $a_{1} \leq a_{2}, b_{1} \leq b_{2}, c_{1} \geq c_{2}$.

Case 4. $a_{1} \leq a_{2}, b_{1} \geq b_{2}, c_{1} \geq c_{2}$.

Case 5. $a_{1} \geq a_{2}, b_{1} \leq b_{2}, c_{1} \leq c_{2}$.

Case 6. $a_{1} \geq a_{2}, b_{1} \geq b_{2}, c_{1} \leq c_{2}$.

Case 7. $a_{1} \geq a_{2}, b_{1} \leq b_{2}, c_{1} \geq c_{2}$.

Case 8. $a_{1} \geq a_{2}, b_{1} \geq b_{2}, c_{1} \geq c_{2}$.

(Insert Figure 1 about here.)

(Insert Table 1 about here.)

\section{Nakamura's Fuzzy Preference Relation}

Using fuzzy minimum, fuzzy maximum and Hamming distance, Nakamura's fuzzy preference relation is defined as follows.

Definition 8. For two fuzzy numbers $A$ and $B$, Nakamura defines $N(A, B)$ and $N^{\prime}(A, B)$ as fuzzy preference relations by the following membership functions

$$
N(A, B)=\frac{d(\underline{A}, \widetilde{m \imath n}(\underline{A}, \underline{B}))+d(\bar{A}, \widetilde{m \imath n}(\bar{A}, \bar{B}))}{d(\underline{A}, \underline{B})+d(\bar{A}, \bar{B})}
$$

and

$$
N^{\prime}(A, B)=\frac{d(A \cap B, 0)+d(\mathrm{~A}, \widetilde{\max }(A, \mathrm{~B}))}{d(A, 0)+d(B, 0)}
$$

respectively. Yuan [6] showed that $N(A, B)$ is reciprocal, transitive and not robust.

Wang and Kerre [3] derived that 


$$
\begin{gathered}
d(\underline{A}, \widetilde{\min }(\underline{A}, \underline{B}))=d(\underline{B}, \widetilde{\max }(\underline{A}, \underline{B})) \\
d(\bar{A}, \widetilde{\max }(\bar{A}, \bar{B}))=d(\bar{B}, \widetilde{\min }(\bar{A}, \bar{B})) \\
d(\underline{A}, \widetilde{\min }(\underline{A}, \underline{B}))+d(\underline{A}, \widetilde{\max }(\underline{A}, \underline{B}))=d(\underline{A}, \underline{B}) \\
d(\bar{A}, \widetilde{\min }(\bar{A}, \bar{B}))+d(\bar{A}, \widetilde{\max }(\bar{A}, \bar{B}))=d(\bar{A}, \bar{B}) \\
2 d(\mathrm{~A} \cap \mathrm{B}, 0)+d(\mathrm{~A}, \widetilde{\max }(A, \mathrm{~B}))+d(\mathrm{~B}, \widetilde{\max }(A, \mathrm{~B}))=d(A, 0)+d(B, 0) .
\end{gathered}
$$

It follows that

$$
\begin{gathered}
N(A, B)+N(B, A)=1 \\
N^{\prime}(A, B)+N^{\prime}(B, A)=1 .
\end{gathered}
$$

For two triangular fuzzy numbers $A\left(a_{1}, b_{1}, c_{1}\right)$ and $B\left(a_{2}, b_{2}, c_{2}\right)$, then

$$
\begin{aligned}
& A_{\alpha}=\left[L_{1}, U_{1}\right]=\left[a_{1}+\left(b_{1}-a_{1}\right) \alpha, c_{1}-\left(c_{1}-b_{1}\right) \alpha\right] \\
& B_{\alpha}=\left[L_{2}, U_{2}\right]=\left[a_{2}+\left(b_{2}-a_{2}\right) \alpha, c_{2}-\left(c_{2}-b_{2}\right) \alpha\right]
\end{aligned}
$$

so

$$
\begin{gathered}
N(A, B)=\frac{d(\underline{A}, \overline{m i n}(\underline{A}, \underline{B}))+d(\bar{A}, \overline{m i n}(\bar{A}, \bar{B}))}{d(\underline{A}, \underline{B})+d(\bar{A}, \bar{B})} \\
=\frac{\int_{L_{1} \geq L_{2}} L_{1}-L_{2} d \alpha+\int_{U_{1} \geq U_{2}} U_{1}-U_{2} d \alpha}{\int_{L_{1} \geq L_{2}} L_{1}-L_{2} d \alpha+\int_{L_{2} \geq L_{1}} L_{2}-L_{1} d \alpha+\int_{U_{1} \geq U_{2}} U_{1}-U_{2} d \alpha+\int_{U_{2} \geq U_{1}} U_{2}-U_{1} d \alpha} .
\end{gathered}
$$

Define

$$
\begin{aligned}
& S_{1}=\int_{L_{1} \geq L_{2}} L_{1}-L_{2} d \alpha=\int_{a_{1}-a_{2}+\left(b_{1}-a_{1}-b_{2}+a_{2}\right) \alpha \geq 0} a_{1}-a_{2}+\left(b_{1}-a_{1}-b_{2}+a_{2}\right) \alpha d \alpha \\
& S_{2}=\int_{L_{2} \geq L_{1}} L_{2}-L_{1} d \alpha=\int_{a_{2}-a_{1}+\left(b_{2}-a_{2}-b_{1}+a_{1}\right) \alpha \geq 0} a_{2}-a_{1}+\left(b_{2}-a_{2}-b_{1}+a_{1}\right) \alpha d \alpha \\
& S_{3}=\int_{U_{1} \geq U_{2}} U_{1}-U_{2} d \alpha=\int_{c_{1}-c_{2}-\left(c_{1}-b_{1}-c_{2}+b_{2}\right) \alpha \geq 0} c_{1}-c_{2}-\left(c_{1}-b_{1}-c_{2}+b_{2}\right) \alpha d \alpha
\end{aligned}
$$


$S_{4}=\int_{U_{2} \geq U_{1}} U_{2}-U_{1} d \alpha=\int_{c_{2}-c_{1}-\left(c_{2}-b_{2}-c_{1}+b_{1}\right) \alpha \geq 0} c_{2}-c_{1}-\left(c_{2}-b_{2}-c_{1}+b_{1}\right) \alpha d \alpha$,

then

$$
N(A, B)=\frac{S_{1}+S_{3}}{S_{1}+S_{2}+S_{3}+S_{4}}
$$

Let $\mathcal{A}=a_{2}-a_{1}, \mathcal{B}=b_{2}-b_{1}$ and $\mathcal{C}=c_{2}-c_{1}$. The steps of implementing the

Nakamura's fuzzy preference relation $N(A, B)$ are as follows.

\section{Algorithm 1.}

If $\mathcal{A} \geq 0$

If $\mathcal{C} \geq 0$

If $\mathcal{B} \geq 0$, then $N(A, B)=0$ else $N(A, B)=\frac{\mathcal{B}^{2}(\mathcal{A}-2 \mathcal{B}+\mathcal{C})}{\left(\mathcal{A}^{2}+\mathcal{B}^{2}\right)(-\mathcal{B}+\mathcal{C})+\left(\mathcal{B}^{2}+\mathcal{C}^{2}\right)(\mathcal{A}-\mathcal{B})}$

else if $\mathcal{B} \geq 0$, then $N(A, B)=\frac{\mathcal{C}^{2}}{(\mathcal{A}+\mathcal{B})(\mathcal{B}-\mathcal{C})+\mathcal{B}^{2}+\mathcal{C}^{2}}$ else

$$
N(A, B)=1-\frac{\mathcal{A}^{2}}{(\mathcal{A}-\mathcal{B})(-\mathcal{B}-\mathcal{C})+\mathcal{A}^{2}+\mathcal{B}^{2}} .
$$

else if $\mathcal{A}<0$

If $\mathcal{C} \geq 0$

If $\mathcal{B} \geq 0$, then $N(A, B)=\frac{\mathcal{A}^{2}}{(-\mathcal{A}+\mathcal{B})(\mathcal{B}+\mathcal{C})+\mathcal{A}^{2}+\mathcal{B}^{2}}$ else

$$
N(A, B)=1-\frac{\mathcal{C}^{2}}{(\mathcal{A}+\mathcal{B})(\mathcal{B}-\mathcal{C})+\mathcal{B}^{2}+\mathcal{C}^{2}}
$$

else if $\mathcal{B} \geq 0$, then $N(A, B)=1-\frac{\mathcal{B}^{2}(\mathcal{A}-2 \mathcal{B}+\mathcal{C})}{\left(\mathcal{A}^{2}+\mathcal{B}^{2}\right)(-\mathcal{B}+\mathcal{C})+\left(\mathcal{B}^{2}+\mathcal{C}^{2}\right)(\mathcal{A}-\mathcal{B})}$ else $N(A, B)=$ 1.

Table 2 shows the values of $N(A, B)$ and $N^{\prime}(A, B)$ for each test case. The first observation of this table is that 


$$
\begin{aligned}
& N_{1}(A, B)+N_{8}(A, B)=1 \\
& N_{2}(A, B)+N_{7}(A, B)=1 \\
& N_{3}(A, B)+N_{6}(A, B)=1 \\
& N_{4}(A, B)+N_{5}(A, B)=1 .
\end{aligned}
$$

Comparing the values of $N(A, B)$ with $N^{\prime}(A, B)$ of each case, we have that $1-$ $N_{1}^{\prime}(A, B) \geq N_{1}(A, B)$ and $1-N_{8}^{\prime}(A, B) \leq N_{8}(A, B)$. If $a_{2}+2 b_{2}+c_{2} \geq a_{1}-2 b_{1}-$ $c_{1}$, we obtain that $1-N_{2}^{\prime}(A, B) \leq N_{2}(A, B), \quad 1-N_{3}^{\prime}(A, B) \geq N_{3}(A, B), \quad 1-$ $N_{4}^{\prime}(A, B) \leq N_{4}(A, B), \quad 1-N_{5}^{\prime}(A, B) \geq N_{5}(A, B), \quad 1-N_{6}^{\prime}(A, B) \leq N_{6}(A, B)$ and $1-$ $N_{7}^{\prime}(A, B) \geq N_{7}(A, B)$.

(Insert Table 2 about here.)

\section{Kołodziejczyk's Fuzzy Preference Relation}

By considering the common part of two membership functions, Kołodziejczyk’s method [5] is based on fuzzy maximum and Hamming distance to propose the following fuzzy preference relation.

Definition 9. For two fuzzy numbers $A$ and $B$, Kołodziejczyk defines $K 1^{\prime}(A, B)$ and $K 2^{\prime}(A, B)$ as fuzzy preference relations by the following membership functions

$$
K 1^{\prime}(A, B)=\frac{d(\underline{A}, \overline{\max }(\underline{A}, \underline{B}))+d(\bar{A}, \overline{\max }(\bar{A}, \bar{B}))+d(A \cap B, 0)}{d(\underline{A}, \underline{B})+d(\bar{A}, \bar{B})+2 d(A \cap B, 0)}
$$

and 


$$
K 2^{\prime}(A, B)=\frac{d(\underline{A}, \widetilde{\max }(\underline{A}, \underline{B}))+d(\bar{A}, \widetilde{\max }(\bar{A}, \bar{B}))}{d(\underline{A}, \underline{B})+d(\bar{A}, \bar{B})}
$$

respectively. $K 1^{\prime}(A, B)$ is reciprocal, transitive and robust $[3,5]$. Since

$$
K 2^{\prime}(A, B)=1-N(A, B)
$$

the results of $K 2^{\prime}(A, B)$ can be obtained from those of $N(A, B)$.

For two triangular fuzzy numbers $A\left(a_{1}, b_{1}, c_{1}\right)$ and $B\left(a_{2}, b_{2}, c_{2}\right)$, then

$$
\begin{aligned}
A_{\alpha} & =\left[L_{1}, U_{1}\right]=\left[a_{1}+\left(b_{1}-a_{1}\right) \alpha, c_{1}-\left(c_{1}-b_{1}\right) \alpha\right] \\
B_{\alpha} & =\left[L_{2}, U_{2}\right]=\left[a_{2}+\left(b_{2}-a_{2}\right) \alpha, c_{2}-\left(c_{2}-b_{2}\right) \alpha\right] .
\end{aligned}
$$

Define

$$
\begin{gathered}
S_{1}=d(\underline{A}, \overline{\max }(\underline{A}, \underline{B}))=\int_{L_{2} \geq L_{1}} L_{2}-L_{1} d \alpha \\
S_{2}=\int_{L_{1} \geq L_{2}} L_{1}-L_{2} d \alpha \\
d(\underline{A}, \underline{B})=S_{1}+S_{2} \\
S_{3}=d(\bar{A}, \overline{\max }(\bar{A}, \bar{B}))=\int_{U_{2} \geq U_{1}} U_{2}-U_{1} d \alpha \\
S_{4}=\int_{U_{1} \geq U_{2}} U_{1}-U_{2} d \alpha \\
d(\bar{A}, \bar{B})=S_{3}+S_{4} \\
S_{5}=d(A \cap B, 0)=\int_{U_{1} \geq L_{2}} U_{1}-L_{2} d \alpha-\int_{U_{1} \geq U_{2}} U_{1}-U_{2} d \alpha-\int_{L_{1} \geq L_{2}} L_{1}-L_{2} d \alpha
\end{gathered}
$$

Then

$$
K 1^{\prime}(A, B)=\frac{S_{1}+S_{3}+S_{5}}{S_{1}+S_{2}+S_{3}+S_{4}+2 S_{5}}
$$

and 


$$
K 2^{\prime}(A, B)=\frac{S_{1}+S_{3}}{S_{1}+S_{2}+S_{3}+S_{4}}
$$

In Table 3, we display the values of $K 1^{\prime}(A, B)$ and $K 2^{\prime}(A, B)$ for each test case. An examination of the table reveals that

$$
\begin{gathered}
K 1_{1}^{\prime}(A, B)=K 1_{3}^{\prime}(A, B)=K 1_{5}^{\prime}(A, B)=K 1_{7}^{\prime}(A, B)=1- \\
\frac{\left(c_{1}-a_{2}\right)^{2}}{\left(c_{1}-a_{2}+c_{2}-a_{1}\right)\left(c_{1}-a_{2}-b_{1}+b_{2}\right)+2\left(b_{2}-b_{1}\right)^{2}} \\
K 1_{2}^{\prime}(A, B)=K 1_{4}^{\prime}(A, B)=K 1_{6}^{\prime}(A, B)=K 1_{8}^{\prime}(A, B)= \\
\frac{\left(c_{2}-a_{1}\right)^{2}}{\left(c_{2}-a_{1}\right)^{2}+\left(c_{2}-a_{1}-b_{2}+b_{1}\right)\left(c_{1}-a_{2}-b_{2}+b_{1}\right)+\left(b_{2}-b_{1}\right)^{2}}
\end{gathered}
$$

If $b_{1}=b_{2}$, we have

$$
\begin{gathered}
K 1_{1}^{\prime}(A, B)=1-\frac{c_{1}-a_{2}}{\left(c_{1}-a_{2}+c_{2}-a_{1}\right)}=\frac{c_{2}-a_{1}}{\left(c_{1}-a_{2}+c_{2}-a_{1}\right)} \\
K 1_{2}^{\prime}(A, B)=\frac{c_{2}-a_{1}}{\left(c_{1}-a_{2}+c_{2}-a_{1}\right)}
\end{gathered}
$$

so

$$
\begin{gathered}
K 1_{1}^{\prime}(A, B)=K 1_{2}^{\prime}(A, B) \\
K 1_{1}^{\prime}(A, B)+K 1_{2}^{\prime}(A, B)=\frac{2\left(c_{2}-a_{1}\right)}{\left(c_{1}-a_{2}+c_{2}-a_{1}\right)} .
\end{gathered}
$$

It follows that

$$
\begin{gathered}
K 1_{1}^{\prime}(A, B)+K 1_{2}^{\prime}(A, B)=0 \text { for } b_{1}=b_{2} \text { and } c_{2}=a_{1} . \\
K 1_{1}^{\prime}(A, B)+K 1_{2}^{\prime}(A, B)=1 \text { for } b_{1}=b_{2} \text { and } c_{1}-a_{2}=c_{2}-a_{1} .
\end{gathered}
$$

(Insert Table 3 about here.) 


\section{Comparative Studies}

If the fuzzy number $A$ is less than the fuzzy number $B$, then the Hamming distance between $A$ and $\widetilde{\max }(A, B)$ is large. Two representations are adopted. One is $\mathrm{d}(A, \widetilde{\max }(A, B))$. The other is $d(\underline{A}, \widetilde{\max }(\underline{A}, \underline{B}))+d(\bar{A}, \widetilde{\max }(\bar{A}, \bar{B}))$ which decompose $A$ into $\bar{A}$ and $\underline{A}$. To analysis the effect of decomposition, we consider the following preference relations without decomposition

$$
T 1^{\prime}(A, B)=\frac{d(A, \widetilde{\max }(A, B))+d(A \cap B, 0)}{d(A, 0)+d(B, 0)}
$$

and

$$
T 2^{\prime}(A, B)=\frac{d(A, \widetilde{\max }(A, B))}{d(A, B)}
$$

which are the counterparts of the Kołodziejczyk's preference relations $K 1^{\prime}(A, B)$ and $K 2^{\prime}(A, B)$. Therefore the preference relations $K 1^{\prime}(A, B)$ and $K 2^{\prime}(A, B)$ consider the decomposition of fuzzy numbers, while $T 1^{\prime}(A, B)$ and $T 2^{\prime}(A, B)$ do not. The preference relations $K 1^{\prime}(A, B)$ and $T 1^{\prime}(A, B)$ consider the intersection of two membership functions, while $K 2^{\prime}(A, B)$ and $T 2^{\prime}(A, B)$ do not. For completeness, Table 4 displays the values of $N(A, B), N^{\prime}(A, B), K 1^{\prime}(A, B), K 2^{\prime}(A, B), T 1^{\prime}(A, B)$ and $T 2^{\prime}(A, B)$ of each test case in terms of the values of $Q_{i}$. The $K 1^{\prime}(A, B)$ considers the decomposition and interection of two fuzzy numbers, while $T 2^{\prime}(A, B)$ do not. From $K 1^{\prime}(A, B)$ to $T 2^{\prime}(A, B)$, two representations are 


$$
K 1^{\prime}(A, B) \rightarrow K 2^{\prime}(A, B) \rightarrow T 2^{\prime}(A, B)
$$

and

$$
K 1^{\prime}(A, B) \rightarrow T 1^{\prime}(A, B) \rightarrow T 2^{\prime}(A, B)
$$

The first feature of Table 4 is that the differences between $K 1^{\prime}(A, B)$ and $T 1^{\prime}(A, B)$ and between $K 2^{\prime}(A, B)$ and $T 2^{\prime}(A, B)$ are $Q_{3}$. More precisely, the numerator and denominator of both $K 1^{\prime}(A, B)$ and $K 2^{\prime}(A, B)$ include $2 Q_{3}$ for cases $1,3,5$ and 7 , the denominator of both $K 1^{\prime}(A, B)$ and $K 2^{\prime}(A, B)$ include $2 Q_{3}$ for cases $2,4,6$ and 8. Therefore, $2 Q_{3}$ represents the effect of the decomposition of fuzzy numbers. The differences between $K 1^{\prime}(A, B)$ and $K 2^{\prime}(A, B)$ and between $T 1^{\prime}(A, B)$ and $T 2^{\prime}(A, B)$ are $Q_{6}$. More precisely, the numerator and denominator of both $K 1^{\prime}(A, B)$ and $T 1^{\prime}(A, B)$ include $Q_{6}$ and $2 Q_{6}$, respectively. Therefore, $Q_{6}$ represents the effect of the intersection of membership fuctions. After some computations, the characteristics of $K 1^{\prime}(A, B), K 2^{\prime}(A, B), T 1^{\prime}(A, B)$ and $T 2^{\prime}(A, B)$ are described as follows.

Theorem 1. Let $T 2^{\prime}(A, B)=\frac{\alpha}{\alpha+\beta}$.

(1) If $b_{1} \leq b_{2}, \beta \leq 2 Q_{3}+\alpha$ or $b_{1} \geq b_{2}, \beta+2 Q_{3} \leq \alpha$, then $K 1^{\prime}(A, B) \leq K 2^{\prime}(A, B)$. If $b_{1} \leq b_{2}, \beta \geq 2 Q_{3}+\alpha$ or $b_{1} \geq b_{2}, \beta+2 Q_{3} \geq \alpha$, then $K 1^{\prime}(A, B) \geq$ $K 2^{\prime}(A, B)$.

(2) If $b_{1} \leq b_{2}$, then $K 2^{\prime}(A, B) \geq T 2^{\prime}(A, B)$. 
If $b_{1} \geq b_{2}$, then $K 2^{\prime}(A, B) \leq T 2^{\prime}(A, B)$.

(3) If $\alpha \geq \beta$, then $T 1^{\prime}(A, B) \leq T 2^{\prime}(A, B)$.

If $\alpha \leq \beta$, then $T 1^{\prime}(A, B) \geq T 2^{\prime}(A, B)$.

(4) If $b_{1} \leq b_{2}$, then $K 1^{\prime}(A, B) \geq T 1^{\prime}(A, B)$.

If $b_{1} \geq b_{2}$, then $K 1^{\prime}(A, B) \leq T 1^{\prime}(A, B)$.

(5) If $b_{1} \leq b_{2}, \beta\left(2 Q_{3}+Q_{6}\right) \leq \alpha Q_{6}$ or $b_{1} \geq b_{2}, \beta Q_{6} \leq \alpha\left(Q_{3}+2 Q_{6}\right)$, then

$K 1^{\prime}(A, B) \leq T 2^{\prime}(A, B)$

If $b_{1} \leq b_{2}, \beta\left(2 Q_{3}+Q_{6}\right) \geq \alpha Q_{6}$ or $b_{1} \geq b_{2}, \beta Q_{6} \geq \alpha\left(Q_{3}+2 Q_{6}\right)$, then

$K 1^{\prime}(A, B) \geq T 2^{\prime}(A, B)$.

For each test case of two triangular fuzzy numbers $A\left(a_{1}, b_{1}, c_{1}\right)$ and $B\left(a_{2}, b_{2}, c_{2}\right)$,

we analyze the behaviors of $K 1^{\prime}(A, B), K 2^{\prime}(A, B), T 1^{\prime}(A, B)$ and $T 2^{\prime}(A, B)$ by applying Theorem 1 as follows. For $b_{1} \leq b_{2}$, we have

$$
T 1^{\prime}(A, B) \leq K 1^{\prime}(A, B) \leq K 2^{\prime}(A, B)=T 2^{\prime}(A, B)=1
$$

for case 1 . For cases 3, 5 and 7, we have

(1) if $a_{2}+2 b_{2}+c_{2} \geq a_{1}+2 b_{1}+c_{1}$, then $K 1^{\prime}(A, B) \leq K 2^{\prime}(A, B)$

if $a_{2}+2 b_{2}+c_{2} \leq a_{1}+2 b_{1}+c_{1}$, then $K 1^{\prime}(A, B) \geq K 2^{\prime}(A, B)$

since $2 Q_{3}+\alpha-\beta=\frac{1}{2}\left(a_{2}+2 b_{2}+c_{2}-a_{1}-2 b_{1}-c_{1}\right)$. 
(2) $K 2^{\prime}(A, B) \geq T 2^{\prime}(A, B)$.

(3) From $\alpha-\beta=\frac{\left(a_{2}-c_{1}\right)\left(a_{2}-b_{1}+b_{2}-c_{1}+c_{2}-a_{1}\right)+\left(b_{1}-b_{2}\right)\left(c_{2}-a_{1}\right)}{2\left(a_{2}+b_{1}-b_{2}-c_{1}\right)}$, it follows that if $a_{2}+b_{2}+c_{2} \geq a_{1}+b_{1}+c_{1}$, then $T 1^{\prime}(A, B) \leq T 2^{\prime}(A, B)$. if $a_{2}+b_{2}+c_{2} \leq a_{1}+b_{1}+c_{1}$, then $T 1^{\prime}(A, B) \geq T 2^{\prime}(A, B)$.

(4) $K 1^{\prime}(A, B) \geq T 1^{\prime}(A, B)$.

(5) If $a_{2}+2 b_{2}+c_{2} \geq a_{1}+2 b_{1}+c_{1}$, then $K 1^{\prime}(A, B) \geq T 2^{\prime}(A, B)$. If $a_{2}+2 b_{2}+c_{2} \leq a_{1}+2 b_{1}+c_{1}$, then $K 1^{\prime}(A, B) \leq T 2^{\prime}(A, B)$.

Therefore, for the cases 3, 5 and 7, if $a_{2}+2 b_{2}+c_{2} \leq a_{1}+2 b_{1}+c_{1}$, then

$$
K 1^{\prime}(A, B) \geq K 2^{\prime}(A, B) \geq T 2^{\prime}(A, B)
$$

and

$$
K 1^{\prime}(A, B) \geq T 1^{\prime}(A, B) \geq T 2^{\prime}(A, B) .
$$

For $b_{1} \geq b_{2}$, we have

$$
K 2^{\prime}(A, B)=T 2^{\prime}(A, B)=0 \leq K 1^{\prime}(A, B) \leq T 1^{\prime}(A, B)
$$

for case 8 . For cases 2, 4 and 6, we have

(1) if $a_{2}+2 b_{2}+c_{2} \geq a_{1}+2 b_{1}+c_{1}$, then $K 1^{\prime}(A, B) \leq K 2^{\prime}(A, B)$

if $a_{2}+2 b_{2}+c_{2} \leq a_{1}+2 b_{1}+c_{1}$, then $K 1^{\prime}(A, B) \geq K 2^{\prime}(A, B)$,

since $\alpha-2 Q_{3}-\beta=\frac{1}{2}\left(a_{2}+2 b_{2}+c_{2}-a_{1}-2 b_{1}-c_{1}\right)$. 
(2) $K 2^{\prime}(A, B) \leq T 2^{\prime}(A, B)$.

(3) From $\alpha-\beta=\frac{1}{2}\left(-a_{1}+a_{2}-2 b_{1}+2 b_{2}-c_{1}+c_{2}+\frac{2\left(b_{1}-b_{2}\right)^{2}}{-a_{1}+b_{1}-b_{2}+c_{2}}\right)$, it follows that if $a_{2}+2 b_{2}+c_{2} \geq a_{1}+2 b_{1}+c_{1}$, then $T 1^{\prime}(A, B) \leq T 2^{\prime}(A, B)$ if $a_{2}+2 b_{2}+c_{2} \leq a_{1}+2 b_{1}+c_{1}$, then $T 1^{\prime}(A, B) \geq T 2^{\prime}(A, B)$.

(4) $K 1^{\prime}(A, B) \leq T 1^{\prime}(A, B)$.

(5) If $a_{2}+2 b_{2}+c_{2} \geq a_{1}+2 b_{1}+c_{1}$, then $K 1^{\prime}(A, B) \leq T 2^{\prime}(A, B)$. If $a_{2}+2 b_{2}+c_{2} \leq a_{1}+2 b_{1}+c_{1}$, then $K 1^{\prime}(A, B) \geq T 2^{\prime}(A, B)$.

Therefore, for the cases 2,4 and 6 , if $a_{2}+2 b_{2}+c_{2} \geq a_{1}+2 b_{1}+c_{1}$, then

$$
K 1^{\prime}(A, B) \leq K 2^{\prime}(A, B) \leq T 2^{\prime}(A, B)
$$

and

$$
K 1^{\prime}(A, B) \leq T 1^{\prime}(A, B) \leq T 2^{\prime}(A, B)
$$

For the two triangular fuzzy numbers $A\left(a_{1}, b_{1}, c_{1}\right)$ and $B\left(a_{2}, b_{2}, c_{2}\right)$, the second comparative study is the five case studies shown in Figure 2 to compare the fuzzy preference relations $K 1^{\prime}(A, B), K 2^{\prime}(A, B), T 1^{\prime}(A, B)$ and $T 2^{\prime}(A, B)$.

$$
\text { (Insert Figure } 2 \text { about here.) }
$$

Case (a) $A\left(a_{1}, b_{1}, c_{1}\right)$ and $B\left(a_{2}, b_{2}, c_{2}\right)$ with $a_{2} \geq c_{1}$.

It follows that 


$$
\begin{gathered}
K 1^{\prime}(A, B)=\frac{\left(Q_{1}+Q_{3}\right)+\left(Q_{2}+Q_{3}\right)+0}{\left(Q_{1}+Q_{3}\right)+\left(Q_{2}+Q_{3}\right)+0}=1 \\
K 2^{\prime}(A, B)=\frac{\left(Q_{1}+Q_{3}\right)+\left(Q_{2}+Q_{3}\right)}{\left(Q_{1}+Q_{3}\right)+\left(Q_{2}+Q_{3}\right)}=1 \\
T 1^{\prime}(A, B)=\frac{0+\left(Q_{1}+Q_{2}\right)}{\left(Q_{1}+Q_{2}\right)}=1 \\
T 2^{\prime}(A, B)=\frac{\left(Q_{1}+Q_{2}\right)}{\left(Q_{1}+Q_{2}\right)}=1 .
\end{gathered}
$$

For this simple case, all the preference relations give the same degree of preference of $B$ over $A$.

Case (b) $A(c-a, c, c+a)$ and $B(c-b, c, c+b)$.

We have

$$
\begin{gathered}
K 1^{\prime}(A, B)=\frac{Q_{1}+0+Q_{2}}{Q_{1}+Q_{3}+2 Q_{2}}=1 / 2 \\
K 2^{\prime}(A, B)=\frac{Q_{1}+0}{Q_{1}+Q_{3}}=1 / 2 \\
T 1^{\prime}(A, B)=\frac{Q_{2}+Q_{1}}{\left(Q_{1}+Q_{2}+Q_{3}\right)+Q_{2}}=1 / 2 . \\
T 2^{\prime}(A, B)=\frac{Q_{1}}{Q_{1}+Q_{3}}=1 / 2
\end{gathered}
$$

From the viewpoint of probability, fuzzy numbers $A$ and $B$ have the same mean, but fuzzy number $B$ with smaller standard deviation. The results indicate that the differences of the decomposition and intersection of $A$ and $B$ cannot affect the degree of preference of $B$ over $A$.

Case (c) $A(a, a+b, a+2 b)$ and $B(a+\alpha, a+b+\alpha+\beta, a+\alpha+2 b+2 \beta)$.

For this case, the fuzzy number $B$ is right shift of the fuzzy number $A$. Therefore, $B$ should have a higher ranking than $A$ based on the intuition criterion. We obtain 


$$
\begin{gathered}
K 1^{\prime}(A, B)=\frac{\left(Q_{2}+Q_{3}\right)+\left(Q_{3}+Q_{4}\right)+Q_{6}}{\left(Q_{2}+Q_{3}\right)+\left(Q_{3}+Q_{4}\right)+2 Q_{6}}=\frac{(2 b+\alpha+2 \beta)^{2}}{2\left(\alpha^{2}+4 b^{2}+4 b \beta+2 b \alpha+2 \beta^{2}\right)}>1 / 2 \\
K 2^{\prime}(A, B)=\frac{\left(Q_{2}+Q_{3}\right)+\left(Q_{3}+Q_{4}\right)}{\left(Q_{2}+Q_{3}\right)+\left(Q_{3}+Q_{4}\right)}=1 \\
T 1^{\prime}(A, B)=\frac{Q_{6}+\left(Q_{2}+Q_{4}\right)}{\left(Q_{2}+Q_{6}\right)+\left(Q_{6}+Q_{4}\right)}=1-\frac{(-2 b+\alpha)^{2}}{2(2 b+\beta)^{2}} \\
T 2^{\prime}(A, B)=\frac{Q_{2}+Q_{4}}{Q_{2}+Q_{4}}=1 .
\end{gathered}
$$

All the methods prefer $B$, but $T 1^{\prime}(A, B)$ is indecisive. More precisely,

If $2 b+\beta<\alpha$, then $T 1^{\prime}(A, B)<1 / 2$, so $A>B$

If $2 b+\beta=\alpha$, then $T 1^{\prime}(A, B)=1 / 2$, so $A=B$

If $2 b+\beta>\alpha$, then $T 1^{\prime}(A, B)>1 / 2$, so $A<B$.

Hence, a conflicting ranking order of $T 1^{\prime}(A, B)$ exists in this case.

Case (d) $A(a, a, a+b)$ and $B(c, c+b, c+b)$ with $a \geq c$.

This case is more complex for the partial overlap of $A$ and $B$. The membership function of $B$ has the right peak, $B$ expands to the left of $A$ for the left membership function, and $A$ expands to the right of $B$ for the right membership function. We have

$$
\begin{gathered}
K 1^{\prime}(A, B)=\frac{\left(Q_{2}+Q_{3}\right)+\left(Q_{3}+Q_{4}\right)+Q_{6}}{\left(Q_{1}+Q_{2}+Q_{3}\right)+\left(Q_{3}+Q_{4}+Q_{5}\right)+2 Q_{6}}=0.5+\frac{b(-2 a+b+2 c)}{a^{2}+3 b^{2}+2 b c+c^{2}-2 a b-2 a c} \\
K 2^{\prime}(A, B)=\frac{\left(Q_{2}+Q_{3}\right)+\left(Q_{3}+Q_{4}\right)}{\left(Q_{1}+Q_{2}+Q_{3}\right)+\left(Q_{3}+Q_{4}+Q_{5}\right)}=\frac{(-a+b+c)^{2}}{2 a^{2}+b^{2}+2 b c+2 c^{2}-2 a b-4 a c} \\
T 1^{\prime}(A, B)=\frac{Q_{6}+\left(Q_{2}+Q_{4}\right)}{\left(Q_{2}+Q_{5}+Q_{6}\right)+\left(Q_{1}+Q_{6}+Q_{4}\right)}=\frac{(a+3 b-c)(-a+b+c)}{4 b^{2}} \\
T 2^{\prime}(A, B)=\frac{Q_{2}+Q_{4}}{Q_{1}+Q_{2}+Q_{4}+Q_{5}}=\frac{(-a+b+c)^{2}}{3 a^{2}+b^{2}+2 b c+3 c^{2}-2 a b-6 a c} .
\end{gathered}
$$

It follows that 
If $-2 a+b+2 c<0$, then $K 1^{\prime}(A, B)<1 / 2$ and $K 2^{\prime}(A, B)<1 / 2$, so $A>B$.

If $-2 a+b+2 c=0$, then $K 1^{\prime}(A, B)=1 / 2$ and $K 2^{\prime}(A, B)=1 / 2$, so $A=B$.

If $-2 a+b+2 c>0$, then $K 1^{\prime}(A, B)>1 / 2$ and $K 2^{\prime}(A, B)>1 / 2$, so $A<B$.

If $b<(1+\sqrt{2})(a-c)$, then $T 1^{\prime}(A, B)<1 / 2$ and $T 2^{\prime}(A, B)<1 / 2$, so $A>B$.

If $b=(1+\sqrt{2})(a-c)$, then $T 1^{\prime}(A, B)=1 / 2$ and $T 2^{\prime}(A, B)=1 / 2$, so $A=B$.

If $b>(1+\sqrt{2})(a-c)$, then $T 1^{\prime}(A, B)>1 / 2$ and $T 2^{\prime}(A, B)>1 / 2$, so $A<B$.

Three special subcases are considered as follows.

(1) Subcase (d1) If $b=(1+\sqrt{2})(a-c)$, then $A(a, a,(2+\sqrt{2}) a-(1+\sqrt{2}) c)$ and $B(c,(1+\sqrt{2}) a-\sqrt{2} c,(1+\sqrt{2}) a-\sqrt{2} c)$, therefore $T 1^{\prime}(A, B)=T 2^{\prime}(A, B)=$ 0.5 , so $A=B$. However, $K 1^{\prime}(A, B)=\frac{6-\sqrt{2}}{8}, K 2^{\prime}(A, B)=2 / 3$ and $A<B$.

(2) Subcase (d2) If $b=2(a-c)$, then $A(a, a, 3 a-2 c)$ and $B(c, 2 a-c, 2 a-c)$, therefore $T 1^{\prime}(A, B)=7 / 16, T 2^{\prime}(A, B)=1 / 3$, so $A>B$. However, $K 1^{\prime}(A, B)=$ $K 2^{\prime}(A, B)=0.5$ and $A=B$.

(3) Subcase (d3) If $A(0.3,0.3,0.9)$ and $B(0.1,0.7,0.7)$, then $K 1^{\prime}(A, B)=0.5556$, $K 2^{\prime}(A, B)=0.6667, T 1^{\prime}(A, B)=0.5556, T 2^{\prime}(A, B)=0.6667$, so $A<B$.

Therefore, if $b<2(a-c)$, then $K 1^{\prime}(A, B)<1 / 2, K 2^{\prime}(A, B)<1 / 2, T 1^{\prime}(A, B)<$ $1 / 2$ and $T 2^{\prime}(A, B)<1 / 2$, so $A>B$, if $b>(1+\sqrt{2})(a-c)$, then $K 1^{\prime}(A, B)>1 / 2$, 
$K 2^{\prime}(A, B)>1 / 2, T 1^{\prime}(A, B)>1 / 2$ and $T 2^{\prime}(A, B)>1 / 2$, so $A<B$.

Case (e) $A(c+a, \mathrm{~b}, 1-c+a)$ and $B(c, 0.5,1-c)$.

For this case, the fuzzy number $B$ is symmetric with respect to $x=0.5$. The fuzzy number $A$ is parallel translation of the fuzzy number $B$ except its peak. We have the following results.

(1) $K 1^{\prime}(A, B)=\frac{\left(Q_{2}+Q_{3}\right)+\left(Q_{3}+Q_{4}\right)+Q_{6}}{\left(Q_{1}+Q_{2}+Q_{3}\right)+\left(Q_{3}+Q_{4}+Q_{5}\right)+2 Q_{6}}=\frac{5-8 b-12 c+8 b c-2 a^{2}+4 b^{2}+8 c^{2}}{7+4 a-8 b-20 c-8 a c+8 b c+4 b^{2}+16 c^{2}} \quad$. If $(1-2 a-2 b)(3+2 a-2 b-4 c)<0$, then $K 1^{\prime}(A, B)<1 / 2$, so $A>B$. For simplicity, the other two conditions are omitted.

(2) $K 2^{\prime}(A, B)=\frac{\left(Q_{2}+Q_{3}\right)+\left(Q_{3}+Q_{4}\right)}{\left(Q_{1}+Q_{2}+Q_{3}\right)+\left(Q_{3}+Q_{4}+Q_{5}\right)}=\frac{(1-2 b)^{2}}{4 a^{2}+(1-2 b)^{2}}$. If $2 a+2 b-1>0$, then $K 2^{\prime}(A, B)<1 / 2$, so $A>B$.

(3) $T 1^{\prime}(A, B)=\frac{Q_{6}+\left(Q_{2}+Q_{4}\right)}{\left(Q_{2}+Q_{5}+Q_{6}\right)+\left(Q_{1}+Q_{6}+Q_{4}\right)}=\frac{2(1-b-c)(1-2 c)-a^{2}}{2(1+2 a-2 b)(3+2 a-2 b-4 c)(1-2 c)} \quad$ and $\quad T 2^{\prime}(A, B)=$ $\frac{Q_{2}+Q_{4}}{Q_{1}+Q_{2}+Q_{4}+Q_{5}}=\frac{(1-2 b)^{2}(1-2 c)}{4 a^{3}+(1-2 b)^{2}(1-2 c)+a^{2}(6-4 b-8 c)} \quad . \quad$ If $\quad a>-0.5+c+$ $\frac{1}{2} \sqrt{(1-2 c)(3-4 b-2 c)}$, then $T 1^{\prime}(A, B)<1 / 2$ and $T 2^{\prime}(A, B)<1 / 2$, so $A>$ B.

Four special cases are considered as follows.

(1) Subcase (e1) If $a=-0.5+c+\frac{1}{2} \sqrt{(1-2 c)(3-4 b-2 c)}$, then $T 1^{\prime}(A, B)=$ $T 2^{\prime}(A, B)=0.5$, so $A=B$. However, $K 1^{\prime}(A, B)>0.5, K 2^{\prime}(A, B)>0.5$ and $A<B$. 
(2) Subcase (e2) If $2 a+2 b-1=0$, then $T 1^{\prime}(A, B)=0.5-\frac{(1-2 b)^{2}}{16(1-b-c)(1-2 c)}<0.5$, $T 2^{\prime}(A, B)=\frac{(1-2 c)}{(3-2 b-4 c)}<0.5$, so $A>B$. However, $K 1^{\prime}(A, B)=K 2^{\prime}(A, B)=0.5$ and $A=B$.

(3) Subcase (e3) If $\mathrm{A}(0.3,0.4,0.9)$ and $\mathrm{B}(0.2,0.5,0.8)$, then $K 1^{\prime}(A, B)=0.4896$, $K 2^{\prime}(A, B)=0.4286, T 1^{\prime}(A, B)=0.4896, T 2^{\prime}(A, B)=0.4286$, so $A>B$.

(4) Subcase (e4) If $b \geq 0.5$, then $(1-2 a-2 b)(3+2 a-2 b-4 c)<0,2 a+2 b-1>0$ and $a>-0.5+c+\frac{1}{2} \sqrt{(1-2 c)(3-4 b-2 c)}$, so $K 1^{\prime}(A, B)<1 / 2, \quad K 2^{\prime}(A, B)<$ $1 / 2, T 1^{\prime}(A, B)<1 / 2$ and $T 2^{\prime}(A, B)<1 / 2$, hence $A>B$.

\section{Conclusion}

This paper analyzes and compares two types of Nakamura's fuzzy preference relations $\left(N(A, B)\right.$ and $\left.N^{\prime}(A, B)\right)$, two types of Kołodziejczyk's fuzzy preference relations $\left(K 1^{\prime}(A, B)\right.$ and $\left.K 2^{\prime}(A, B)\right)$ and the counterparts of the Kołodziejczyk's fuzzy preference relations $\left(T 1^{\prime}(A, B)\right.$ and $\left.T 2^{\prime}(A, B)\right)$ on a group of selected eight cases with different level of overlapping between two triangular fuzzy numbers $\left(a_{1}, b_{1}, c_{1}\right)$ and $B\left(a_{2}, b_{2}, c_{2}\right)$. For $N(A, B)$ and $N^{\prime}(A, B), N_{j}(A, B)+N_{8-j}(A, B)=1, j=$ $1,2,3,4$. If $a_{2}+2 b_{2}+c_{2} \geq a_{1}-2 b_{1}-c_{1}$, we have that $1-N_{j}^{\prime}(A, B) \geq N_{j}(A, B)$ for $j=1,3,5,7$ and $1-N_{j}^{\prime}(A, B) \leq N_{j}(A, B)$ for $j=2,4,6,8$. For $K 1^{\prime}(A, B)$ and $K 2^{\prime}(A, B), \quad K 1_{1}^{\prime}(A, B)=K 1_{j}^{\prime}(A, B)$ for $j=3,5,7$ and $K 1_{2}^{\prime}(A, B)=K 1_{j}^{\prime}(A, B)$ for 
$j=4,6,8$. Furthermore, $K 1_{1}^{\prime}(A, B)+K 1_{2}^{\prime}(A, B)=0$ for $b_{1}=b_{2}$ and $c_{2}=a_{1}$ and $K 1_{1}^{\prime}(A, B)+K 1_{2}^{\prime}(A, B)=1$ for $b_{1}=b_{2}$ and $c_{1}-a_{2}=c_{2}-a_{1}$. For test case 1 , $T 1^{\prime}(A, B) \leq K 1^{\prime}(A, B) \leq K 2^{\prime}(A, B)=T 2^{\prime}(A, B)=1$. For the test cases 3,5 and 7 , if $a_{2}+2 b_{2}+c_{2} \leq a_{1}+2 b_{1}+c_{1} \quad, \quad$ then $\quad K 1^{\prime}(A, B) \geq K 2^{\prime}(A, B) \geq T 2^{\prime}(A, B) \quad$ and $K 1^{\prime}(A, B) \geq T 1^{\prime}(A, B) \geq T 2^{\prime}(A, B)$. For test case 8 , we have $K 2^{\prime}(A, B)=T 2^{\prime}(A, B)=$ $0 \leq K 1^{\prime}(A, B) \leq T 1^{\prime}(A, B)$. For test cases 2,4 and 6 , if $a_{2}+2 b_{2}+c_{2} \geq a_{1}+2 b_{1}+$ $c_{1}$, then $K 1^{\prime}(A, B) \leq K 2^{\prime}(A, B) \leq T 2^{\prime}(A, B)$ and $K 1^{\prime}(A, B) \leq T 1^{\prime}(A, B) \leq T 2^{\prime}(A, B)$.

\section{References}

1. Chen, S.J.; Hwang, C.L., Fuzzy Multiple Attribute Decision Making, Springer, Berlin, 1992.

2. Wang, X.; Kerre, E.E. Reasonable properties for the ordering of fuzzy quantities (I). Fuzzy Sets Syst.2001, 118, 375-385.

3. Wang, X.; Kerre, E.E. Reasonable properties for the ordering of fuzzy quantities (II). Fuzzy Sets Syst.2001, 118, 387-405.

4. Nakamura, K. Preference relations on a set of fuzzy utilities as a basis for decision making. Fuzzy Sets Syst.1986, 20, 147-162.

5 Kolodziejczyk W. Orlovsky's concept of decision-making with fuzzy preference relation- further results. Fuzzy Sets Syst.1990, 19, 197-212. 
6. Yuan, Y. Criteria for evaluating fuzzy ranking methods. Fuzzy Sets Syst. 1991, 44, 139-157.

7. Li, R.J. Fuzzy method in group decision making. Comput. Math. Appl. 1999, 38, $91-101$.

8. Lee, H.S. On fuzzy preference relation in group decision making. Int. J. Comput. Math. 2005, 82, 133-140.

9. Asady, B. The revised method of ranking LR fuzzy number based on deviation degree. Expert Syst. Appl. 2010, 37, 5056-5060.

10. Wang, Z.X.; Liu, Y.J.; Fan, Z.P.; Feng, B. Ranking LR fuzzy number based on deviation degree. Inf. Sci. 2009, 179, 2070-2077.

11. Zhang, F.; Ignatius, J.; Lim, C.P.; Zhao, Y. A new method for ranking fuzzy numbers and its application to group decision making. Appl. Math. Model. 2014, $38,1563-1582$.

12. Zhu, B.; Xu, Z.; Xu, J. Deriving a ranking from hesitant fuzzy preference relations under group decision making. IEEE Tran. Cyber. 2014, 44, 1328-1337.

13. Wang, Y.J. Ranking triangle and trapezoidal fuzzy numbers based on the relative preference relation. Appl. Math. Model. 2015, 39, 586-599. 
14. Liu X.; Wang Z.; Zhang S. A modification on the hesitant fuzzy set lexicographical ranking method. Symmetry 2016, 8, doi:10.3390/sym8120153.

15. Farhadinia, B. Hesitant fuzzy set lexicographical ordering and its application to multi-attribute decision making. Inf. Sci. 2016, 327, 233-245. 
Case 1. $a_{1} \leq a_{2}, b_{1} \leq b_{2}, c_{1} \leq c_{2}$.

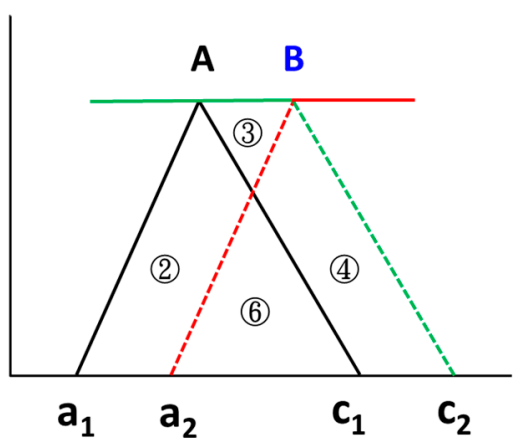

Case 2. $a_{1} \leq a_{2}, b_{1} \geq b_{2}, c_{1} \leq c_{2}$.

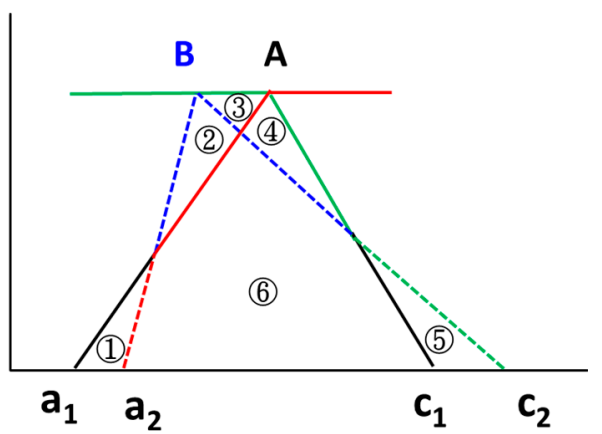

Case 3. $a_{1} \leq a_{2}, b_{1} \leq b_{2}, c_{1} \geq c_{2}$.

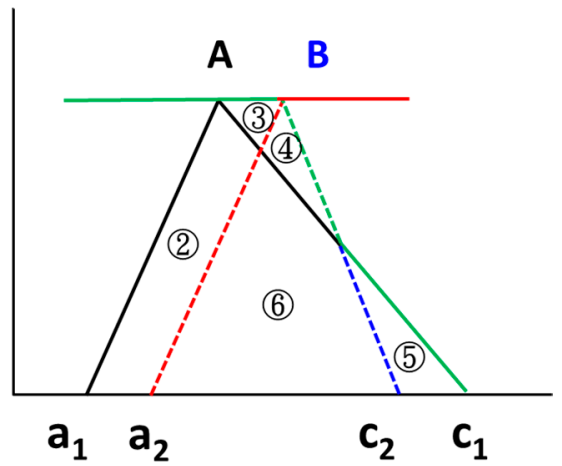

Case 4. $a_{1} \leq a_{2}, b_{1} \geq b_{2}, c_{1} \geq c_{2}$.

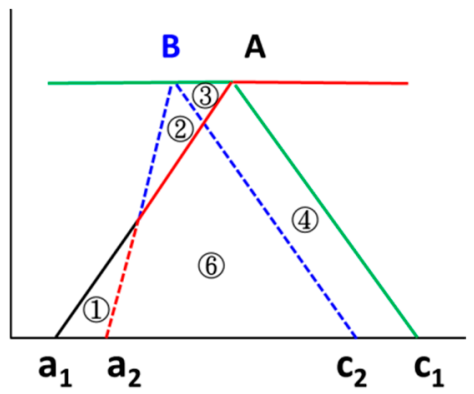

Case 5. $a_{1} \geq a_{2}, b_{1} \leq b_{2}, c_{1} \leq c_{2}$. 


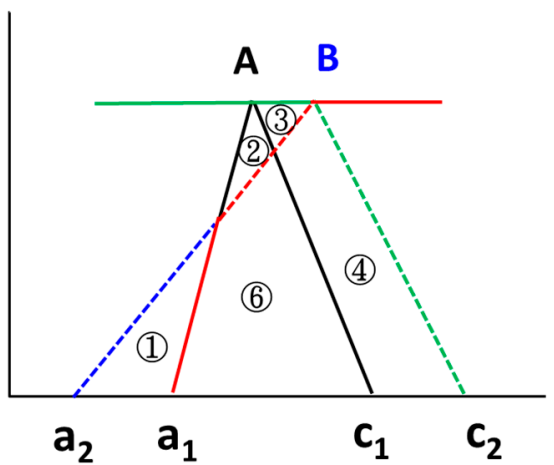

Case 6. $a_{1} \geq a_{2}, b_{1} \geq b_{2}, c_{1} \leq c_{2}$.

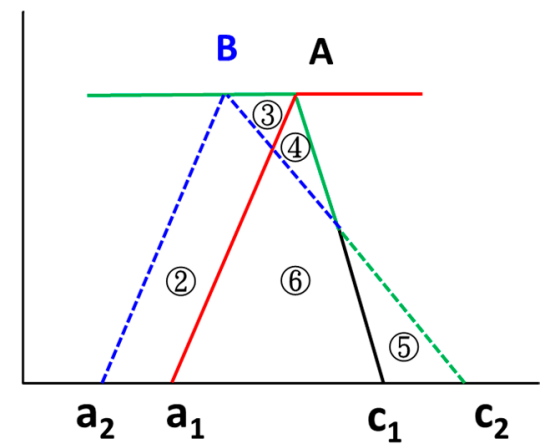

Case 7. $a_{1} \geq a_{2}, b_{1} \leq b_{2}, c_{1} \geq c_{2}$.

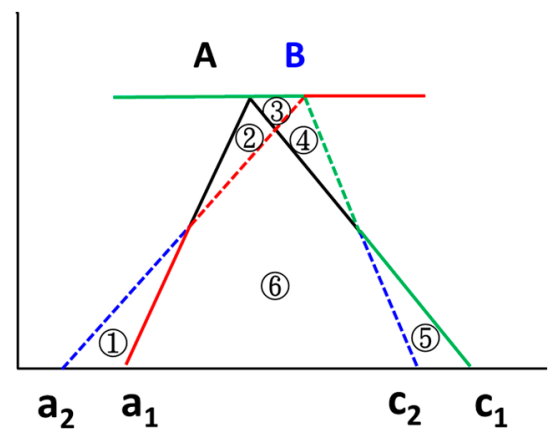

Case 8. $a_{1} \geq a_{2}, b_{1} \geq b_{2}, c_{1} \geq c_{2}$.

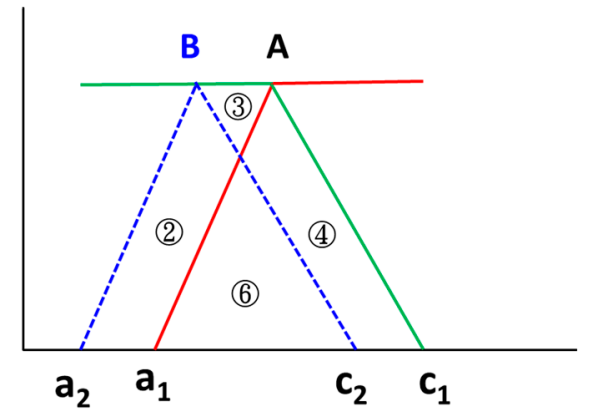

Figure 1. Eight test cases for two fuzzy numbers $A\left(a_{1}, b_{1}, c_{1}\right)$ and $B\left(a_{2}, b_{2}, c_{2}\right)$. 
Case (a) $A\left(a_{1}, b_{1}, c_{1}\right)$ and $B\left(a_{2}, b_{2}, c_{2}\right)$ with $a_{2} \geq c_{1}$

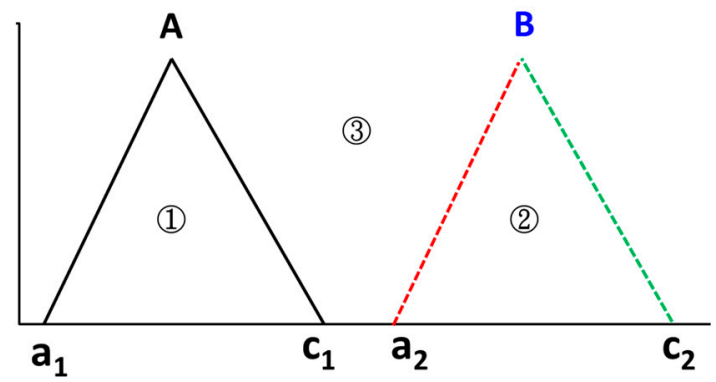

Case (b) $A(c-a, c, c+a)$ and $B(c-b, c, c+b)$.

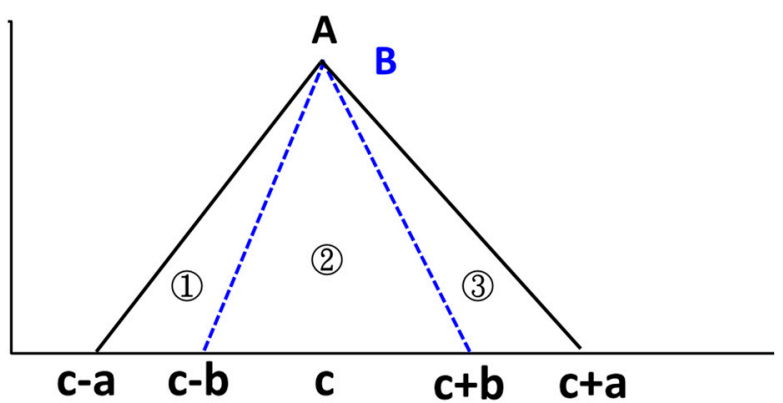

Case (c) $A(a, a+b, a+2 b)$ and $B(a+\alpha, a+b+\alpha+\beta, a+\alpha+2 b+2 \beta)$.

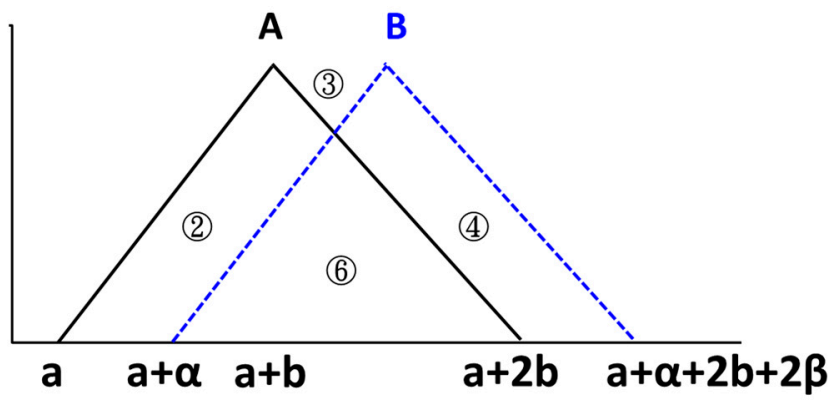

Case (d) $\mathrm{A}(a, a, a+b)$ and $\mathrm{B}(c, c+b, c+b)$.

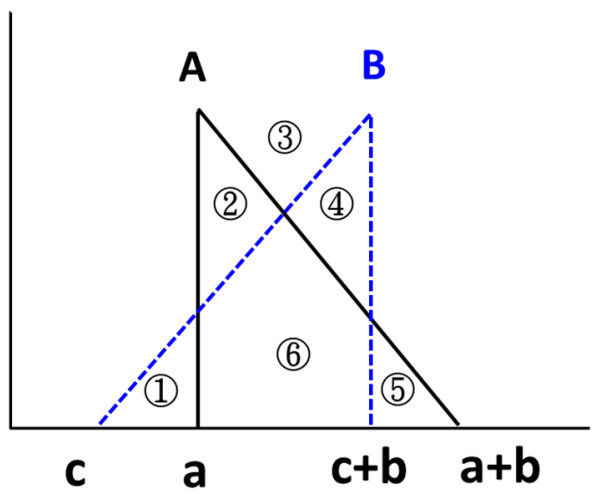


Case (e) $A(c+a, \mathrm{~b}, 1-c+a)$ and $B(c, 0.5,1-c)$.

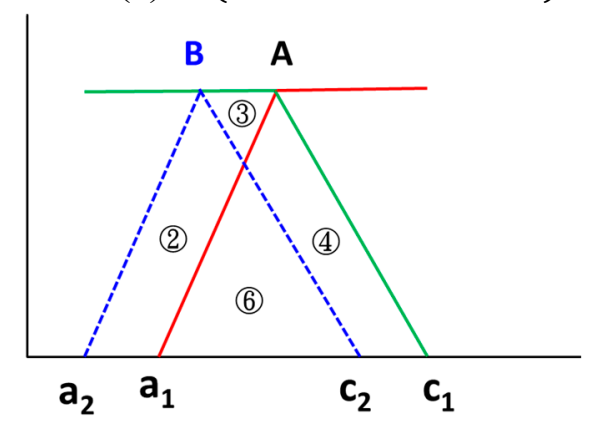

Figure 2. Five case studies of $A$ and $B$ for $K 1^{\prime}(A, B), K 2^{\prime}(A, B), T 1^{\prime}(A, B)$ and $T 2^{\prime}(A, B)$. 
Table 1. The area $Q_{i}$ of $i$-th region for eight cases.

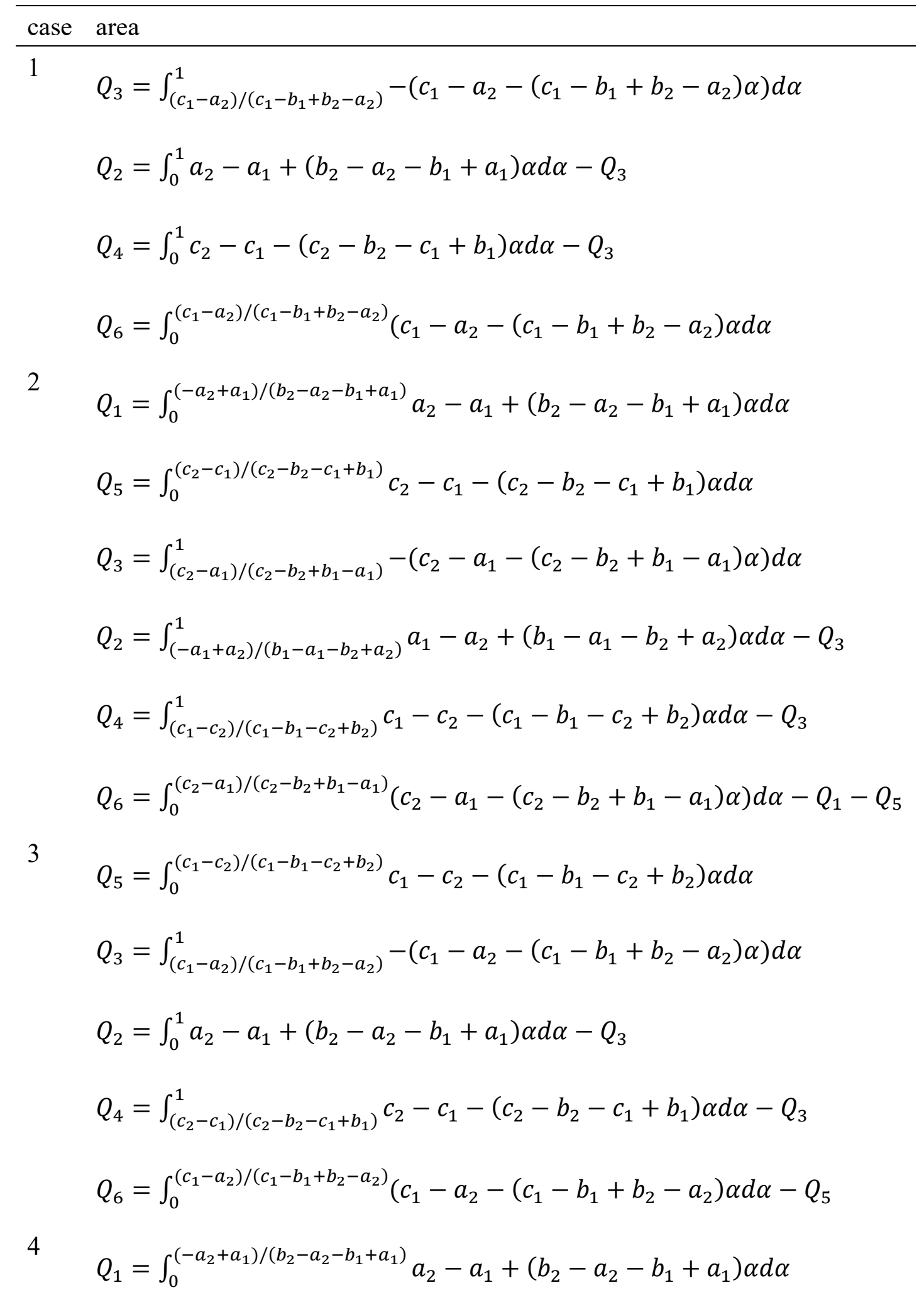




$$
\begin{aligned}
& Q_{3}=\int_{\left(c_{2}-a_{1}\right) /\left(c_{2}-b_{2}+b_{1}-a_{1}\right)}^{1}-\left(c_{2}-a_{1}-\left(c_{2}-b_{2}+b_{1}-a_{1}\right) \alpha\right) d \alpha \\
& Q_{2}=\int_{\left(-a_{1}+a_{2}\right) /\left(b_{1}-a_{1}-b_{2}+a_{2}\right)}^{1} a_{1}-a_{2}+\left(b_{1}-a_{1}-b_{2}+a_{2}\right) \alpha d \alpha-Q_{3} \\
& Q_{4}=\int_{0}^{1} c_{1}-c_{2}-\left(c_{1}-b_{1}-c_{2}+b_{2}\right) \alpha d \alpha-Q_{3} \\
& Q_{6}=\int_{0}^{\left(c_{2}-a_{1}\right) /\left(c_{2}-b_{2}+b_{1}-a_{1}\right)}\left(c_{2}-a_{1}-\left(c_{2}-b_{2}+b_{1}-a_{1}\right) \alpha\right) d \alpha-Q_{1}
\end{aligned}
$$

$$
\begin{aligned}
& Q_{1}=\int_{0}^{\left(-a_{1}+a_{2}\right) /\left(b_{1}-a_{1}-b_{2}+a_{2}\right)} a_{1}-a_{2}+\left(b_{1}-a_{1}-b_{2}+a_{2}\right) \alpha d \alpha \\
& Q_{3}=\int_{\left(c_{1}-a_{2}\right) /\left(c_{1}-b_{1}+b_{2}-a_{2}\right)}^{1}-\left(c_{1}-a_{2}-\left(c_{1}-b_{1}+b_{2}-a_{2}\right) \alpha\right) d \alpha \\
& Q_{2}=\int_{\left(-a_{2}+a_{1}\right) /\left(b_{2}-a_{2}-b_{1}+a_{1}\right)}^{1} a_{2}-a_{1}+\left(b_{2}-a_{2}-b_{1}+a_{1}\right) \alpha d \alpha-Q_{3} \\
& Q_{4}=\int_{0}^{1} c_{2}-c_{1}-\left(c_{2}-b_{2}-c_{1}+b_{1}\right) \alpha d \alpha-Q_{3} \\
& Q_{6}=\int_{0}^{\left(c_{1}-a_{2}\right) /\left(c_{1}-b_{1}+b_{2}-a_{2}\right)}\left(c_{1}-a_{2}-\left(c_{1}-b_{1}+b_{2}-a_{2}\right) \alpha d \alpha-Q_{1}\right.
\end{aligned}
$$

6

$$
\begin{aligned}
& Q_{5}=\int_{0}^{\left(c_{2}-c_{1}\right) /\left(c_{2}-b_{2}-c_{1}+b_{1}\right)} c_{2}-c_{1}-\left(c_{2}-b_{2}-c_{1}+b_{1}\right) \alpha d \alpha \\
& Q_{3}=\int_{\left(c_{2}-a_{1}\right) /\left(c_{2}-b_{2}+b_{1}-a_{1}\right)}^{1}-\left(c_{2}-a_{1}-\left(c_{2}-b_{2}+b_{1}-a_{1}\right) \alpha\right) d \alpha \\
& Q_{2}=\int_{0}^{1} a_{1}-a_{2}+\left(b_{1}-a_{1}-b_{2}+a_{2}\right) \alpha d \alpha-Q_{3} \\
& Q_{4}=\int_{\left(c_{1}-c_{2}\right) /\left(c_{1}-b_{1}-c_{2}+b_{2}\right)}^{1} c_{1}-c_{2}-\left(c_{1}-b_{1}-c_{2}+b_{2}\right) \alpha d \alpha-Q_{3} \\
& Q_{6}=\int_{0}^{\left(c_{2}-a_{1}\right) /\left(c_{2}-b_{2}+b_{1}-a_{1}\right)}\left(c_{2}-a_{1}-\left(c_{2}-b_{2}+b_{1}-a_{1}\right) \alpha\right) d \alpha-Q_{5}
\end{aligned}
$$

7

$$
\begin{aligned}
& Q_{1}=\int_{0}^{\left(-a_{1}+a_{2}\right) /\left(b_{1}-a_{1}-b_{2}+a_{2}\right)} a_{1}-a_{2}+\left(b_{1}-a_{1}-b_{2}+a_{2}\right) \alpha d \alpha \\
& Q_{5}=\int_{0}^{\left(c_{1}-c_{2}\right) /\left(c_{1}-b_{1}-c_{2}+b_{2}\right)} c_{1}-c_{2}-\left(c_{1}-b_{1}-c_{2}+b_{2}\right) \alpha d \alpha \\
& Q_{3}=\int_{\left(c_{1}-a_{2}\right) /\left(c_{1}-b_{1}+b_{2}-a_{2}\right)}^{1}-\left(c_{1}-a_{2}-\left(c_{1}-b_{1}+b_{2}-a_{2}\right) \alpha\right) d \alpha \\
& Q_{2}=\int_{\left(-a_{2}+a_{1}\right) /\left(b_{2}-a_{2}-b_{1}+a_{1}\right)}^{1} a_{2}-a_{1}+\left(b_{2}-a_{2}-b_{1}+a_{1}\right) \alpha d \alpha-Q_{3}
\end{aligned}
$$




$$
\begin{aligned}
& Q_{4}=\int_{\left(c_{2}-c_{1}\right) /\left(c_{2}-b_{2}-c_{1}+b_{1}\right)}^{1} c_{2}-c_{1}-\left(c_{2}-b_{2}-c_{1}+b_{1}\right) \alpha d \alpha-Q_{3} \\
& Q_{6}=\int_{0}^{\left(c_{1}-a_{2}\right) /\left(c_{1}-b_{1}+b_{2}-a_{2}\right)}\left(c_{1}-a_{2}-\left(c_{1}-b_{1}+b_{2}-a_{2}\right) \alpha d \alpha-Q_{1}-Q_{5}\right. \\
& Q_{3}=\int_{\left(c_{2}-a_{1}\right) /\left(c_{2}-b_{2}+b_{1}-a_{1}\right)}^{1}-\left(c_{2}-a_{1}-\left(c_{2}-b_{2}+b_{1}-a_{1}\right) \alpha\right) d \alpha \\
& Q_{2}=\int_{0}^{1} a_{1}-a_{2}+\left(b_{1}-a_{1}-b_{2}+a_{2}\right) \alpha d \alpha-Q_{3} \\
& Q_{4}=\int_{0}^{1} c_{1}-c_{2}-\left(c_{1}-b_{1}-c_{2}+b_{2}\right) \alpha d \alpha-Q_{3} \\
& Q_{6}=\int_{0}^{\left(c_{2}-a_{1}\right) /\left(c_{2}-b_{2}+b_{1}-a_{1}\right)}\left(c_{2}-a_{1}-\left(c_{2}-b_{2}+b_{1}-a_{1}\right) \alpha\right) d \alpha
\end{aligned}
$$$$
8
$$ 
Table 2. $N(A, B)$ and $N^{\prime}(A, B)$ for eight cases.

\begin{tabular}{|c|c|c|}
\hline case & $N(A, B)$ & $N^{\prime}(A, B)$ \\
\hline 1 & 0 & $1+\frac{\left(a_{2}-c_{1}\right)^{2}}{\left(a_{2}+b_{1}-b_{2}-c_{1}\right)\left(-a_{1}-a_{2}+c_{1}+c_{2}\right)}$ \\
\hline \multirow[t]{2}{*}{2} & $\left(b_{2}-b_{1}\right)^{2}\left(a_{2}-a_{1}-2\left(b_{2}-b_{1}\right)+\left(c_{2}-c_{1}\right)\right)$ & $\left(a_{1}-c_{2}\right)^{2}$ \\
\hline & $\overline{\left(\left(a_{2}-a_{1}\right)^{2}+\left(b_{2}-b_{1}\right)^{2}\right)\left(b_{1}-b_{2}+c_{2}-c_{1}\right)+\left(\left(b_{2}-b_{1}\right)^{2}+\left(c_{2}-c_{1}\right)^{2}\right)\left(a_{2}-a_{1}-b_{2}+b_{1}\right)}$ & $\overline{\left(a_{1}-b_{1}+b_{2}-c_{2}\right)\left(a_{1}+a_{2}-c_{1}-c_{2}\right)}$ \\
\hline \multirow[t]{2}{*}{3} & $\left(c_{2}-c_{1}\right)^{2}$ & $1+\frac{\left(a_{2}-c_{1}\right)^{2}}{}$ \\
\hline & $\left(a_{2}-a_{1}+b_{2}-b_{1}\right)\left(b_{2}-b_{1}-c_{2}+c_{1}\right)+\left(b_{2}-b_{1}\right)^{2}+\left(c_{2}-c_{1}\right)^{2}$ & $\left(a_{2}+b_{1}-b_{2}-c_{1}\right)\left(-a_{1}-a_{2}+c_{1}+c_{2}\right)$ \\
\hline \multirow[t]{2}{*}{4} & $1-\frac{\left(a_{2}-a_{1}\right)^{2}}{}$ & $\left(a_{1}-c_{2}\right)^{2}$ \\
\hline & $\left(a_{2}-a_{1}-b_{2}+b_{1}\right)\left(-b_{2}+b_{1}-c_{2}+c_{1}\right)+\left(a_{2}-a_{1}\right)^{2}+\left(b_{2}-b_{1}\right)^{2}$ & $\left(a_{1}-b_{1}+b_{2}-c_{2}\right)\left(a_{1}+a_{2}-c_{1}-c_{2}\right)$ \\
\hline \multirow[t]{2}{*}{5} & $\left(a_{2}-a_{1}\right)^{2}$ & $1+\frac{\left(a_{2}-c_{1}\right)^{2}}{}$ \\
\hline & $\left(a_{2}-a_{1}-b_{2}+b_{1}\right)\left(-b_{2}+b_{1}-c_{2}+c_{1}\right)+\left(a_{2}-a_{1}\right)^{2}+\left(b_{2}-b_{1}\right)^{2}$ & $\left(a_{2}+b_{1}-b_{2}-c_{1}\right)\left(-a_{1}-a_{2}+c_{1}+c_{2}\right)$ \\
\hline \multirow[t]{2}{*}{6} & $1-\frac{\left(c_{2}-c_{1}\right)^{2}}{-}$ & $\left(a_{1}-c_{2}\right)^{2}$ \\
\hline & $\overline{\left(a_{2}-a_{1}+b_{2}-b_{1}\right)\left(b_{2}-b_{1}-c_{2}+c_{1}\right)+\left(b_{2}-b_{1}\right)^{2}+\left(c_{2}-c_{1}\right)^{2}}$ & $\left(a_{1}-b_{1}+b_{2}-c_{2}\right)\left(a_{1}+a_{2}-c_{1}-c_{2}\right)$ \\
\hline \multirow[t]{2}{*}{7} & $\left(b_{2}-b_{1}\right)^{2}\left(a_{2}-a_{1}-2 b_{2}+2 b_{1}+c_{2}-c_{1}\right)$ & $\left(a_{2}-c_{1}\right)^{2}$ \\
\hline & $\overline{\left(\left(a_{2}-a_{1}\right)^{2}+\left(b_{2}-b_{1}\right)^{2}\right)\left(b_{1}-b_{2}+c_{2}-c_{1}\right)+\left(\left(b_{2}-b_{1}\right)^{2}+\left(c_{2}-c_{1}\right)^{2}\right)\left(a_{2}-a_{1}-b_{2}+b_{1}\right)}$ & $\top \overline{\left(a_{2}+b_{1}-b_{2}-c_{1}\right)\left(-a_{1}-a_{2}+c_{1}+c_{2}\right)}$ \\
\hline \multirow[t]{2}{*}{8} & 1 & $\left(a_{1}-c_{2}\right)^{2}$ \\
\hline & & $\overline{\left(a_{1}-b_{1}+b_{2}-c_{2}\right)\left(a_{1}+a_{2}-c_{1}-c_{2}\right)}$ \\
\hline
\end{tabular}


Table 3. $K 1^{\prime}(A, B)$ and $K 2^{\prime}(A, B)$ for eight cases.

\begin{tabular}{|c|c|c|}
\hline case & $K 1^{\prime}(A, B)$ & $K 2^{\prime}(A, B)$ \\
\hline 1 & $1-\frac{\left(c_{1}-a_{2}\right)^{2}}{\left(c_{1}-a_{2}+c_{2}-a_{1}\right)\left(c_{1}-a_{2}-b_{1}+b_{2}\right)+2\left(b_{2}-b_{1}\right)^{2}}$ & 1 \\
\hline \multirow[t]{2}{*}{2} & $\left(c_{2}-a_{1}\right)^{2}$ & $\left(b_{2}-b_{1}\right)^{2}\left(a_{2}-a_{1}-2\left(b_{2}-b_{1}\right)+\left(c_{2}-c_{1}\right)\right)$ \\
\hline & $\overline{\left(c_{2}-a_{1}\right)^{2}+\left(c_{2}-a_{1}-b_{2}+b_{1}\right)\left(c_{1}-a_{2}-b_{2}+b_{1}\right)+\left(b_{2}-b_{1}\right)^{2}}$ & $1-\overline{\left(\left(a_{2}-a_{1}\right)^{2}+\left(b_{2}-b_{1}\right)^{2}\right)\left(b_{1}-b_{2}+c_{2}-c_{1}\right)+\left(\left(b_{2}-b_{1}\right)^{2}+\left(c_{2}-c_{1}\right)^{2}\right)\left(a_{2}-a_{1}-b_{2}+b_{1}\right)}$ \\
\hline 3 & $1-\frac{\left(c_{1}-a_{2}\right)^{2}}{\left(c_{1}-a_{2}+c_{2}-a_{1}\right)\left(c_{1}-a_{2}-b_{1}+b_{2}\right)+2\left(b_{2}-b_{1}\right)^{2}}$ & $1-\frac{\left(c_{2}-c_{1}\right)^{2}}{\left(a_{2}-a_{1}+b_{2}-b_{1}\right)\left(b_{2}-b_{1}-c_{2}+c_{1}\right)+\left(b_{2}-b_{1}\right)^{2}+\left(c_{2}-c_{1}\right)^{2}}$ \\
\hline \multirow[t]{2}{*}{4} & $\left(c_{2}-a_{1}\right)^{2}$ & $\left(a_{2}-a_{1}\right)^{2}$ \\
\hline & $\overline{\left(c_{2}-a_{1}\right)^{2}+\left(c_{2}-a_{1}-b_{2}+b_{1}\right)\left(c_{1}-a_{2}-b_{2}+b_{1}\right)+\left(b_{2}-b_{1}\right)^{2}}$ & $\overline{\left(a_{2}-a_{1}-b_{2}+b_{1}\right)\left(-b_{2}+b_{1}-c_{2}+c_{1}\right)+\left(a_{2}-a_{1}\right)^{2}+\left(b_{2}-b_{1}\right)^{2}}$ \\
\hline 5 & $1-\frac{\left(c_{1}-a_{2}\right)^{2}}{\left(c_{1}-a_{2}+c_{2}-a_{1}\right)\left(c_{1}-a_{2}-b_{1}+b_{2}\right)+2\left(b_{2}-b_{1}\right)^{2}}$ & $1-\frac{\left(a_{2}-a_{1}\right)^{2}}{\left(a_{2}-a_{1}-b_{2}+b_{1}\right)\left(-b_{2}+b_{1}-c_{2}+c_{1}\right)+\left(a_{2}-a_{1}\right)^{2}+\left(b_{2}-b_{1}\right)^{2}}$ \\
\hline \multirow[t]{2}{*}{6} & $\left(c_{2}-a_{1}\right)^{2}$ & $\left(c_{2}-c_{1}\right)^{2}$ \\
\hline & $\overline{\left(c_{2}-a_{1}\right)^{2}+\left(c_{2}-a_{1}-b_{2}+b_{1}\right)\left(c_{1}-a_{2}-b_{2}+b_{1}\right)+\left(b_{2}-b_{1}\right)^{2}}$ & $\overline{\left(a_{2}-a_{1}+b_{2}-b_{1}\right)\left(b_{2}-b_{1}-c_{2}+c_{1}\right)+\left(b_{2}-b_{1}\right)^{2}+\left(c_{2}-c_{1}\right)^{2}}$ \\
\hline \multirow[t]{2}{*}{7} & $1-\frac{\left(c_{1}-a_{2}\right)^{2}}{-}$ & $\left(b_{2}-b_{1}\right)^{2}\left(a_{2}-a_{1}-2 b_{2}+2 b_{1}+c_{2}-c_{1}\right)$ \\
\hline & $1-\overline{\left(c_{1}-a_{2}+c_{2}-a_{1}\right)\left(c_{1}-a_{2}-b_{1}+b_{2}\right)+2\left(b_{2}-b_{1}\right)^{2}}$ & $\overline{\left(\left(a_{2}-a_{1}\right)^{2}+\left(b_{2}-b_{1}\right)^{2}\right)\left(b_{1}-b_{2}+c_{2}-c_{1}\right)+\left(\left(b_{2}-b_{1}\right)^{2}+\left(c_{2}-c_{1}\right)^{2}\right)\left(a_{2}-a_{1}-b_{2}+b_{1}\right)}$ \\
\hline \multirow[t]{2}{*}{8} & $\left(c_{2}-a_{1}\right)^{2}$ & 0 \\
\hline & $\overline{\left(c_{2}-a_{1}\right)^{2}+\left(c_{2}-a_{1}-b_{2}+b_{1}\right)\left(c_{1}-a_{2}-b_{2}+b_{1}\right)+\left(b_{2}-b_{1}\right)^{2}}$ & \\
\hline
\end{tabular}


Table 4. $N(A, B), N^{\prime}(A, B), K 1^{\prime}(A, B), K 2^{\prime}(A, B), T 1^{\prime}(A, B)$ and $T 2^{\prime}(A, B)$ for eight cases.

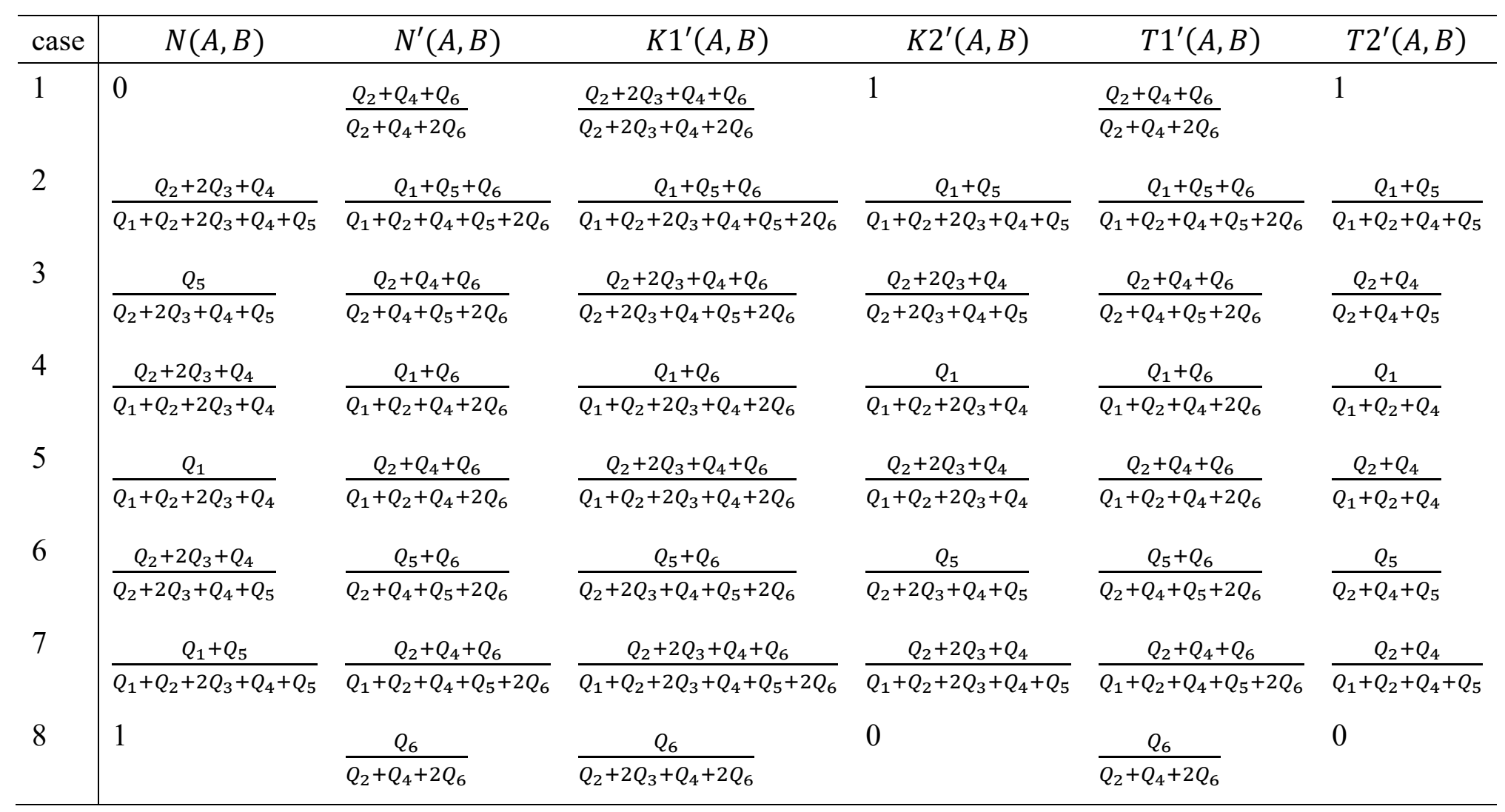

